TRANSACTIONS OF THE

AMERICAN MATHEMATICAL SOCIETY

Volume 355, Number 1, Pages 241-263

S 0002-9947(02)03055-6

Article electronically published on August 28, 2002

\title{
ANOTHER WAY TO SAY HARMONIC
}

\author{
MICHAEL G. CRANDALL AND JIANYING ZHANG
}

\begin{abstract}
It is known that solutions of $-\Delta_{\infty} u=-\sum_{i, j=1}^{n} u_{x_{i}} u_{x_{j}} u_{x_{i} x_{j}}=$ 0 , that is, the $\infty$-harmonic functions, are exactly those functions having a comparison property with respect to the family of translates of the radial solutions $G(x)=a|x|$. We establish a more difficult linear result: a function in $\mathbb{R}^{n}$ is harmonic if it has the comparison property with respect to sums of $n$ translates of the radial harmonic functions $G(x)=a|x|^{2-n}$ for $n \neq 2$ and $G(x)=b \ln (|x|)$ for $n=2$. An attempt to generalize these results for $-\Delta_{\infty} u=0(p=\infty)$ and $-\Delta u=0(p=2)$ to the general $p$-Laplacian leads to the fascinating discovery that certain sums of translates of radial $p$ superharmonic functions are again $p$-superharmonic. Mystery remains: the class of $p$-superharmonic functions so constructed for $p \notin\{2, \infty\}$ does not suffice to characterize $p$-subharmonic functions.
\end{abstract}

\section{INTRODUCTION}

The infinity Laplacian $\Delta_{\infty}$ on $\mathbb{R}^{n}$ is defined by the formal expression

$$
-\Delta_{\infty} u=-\sum_{i, j=1}^{n} u_{x_{i}} u_{x_{j}} u_{x_{i} x_{j}} .
$$

The set of "cone functions",

$$
\mathcal{C}_{\infty}=\left\{x \mapsto a|x-z|: a \in \mathbb{R}^{+}, z \in \mathbb{R}^{n}\right\},
$$

is the set of translates of nonnegative radial solutions of $-\Delta_{\infty} u=0$ if one excludes the vertices. It was observed in 4 that an upper-semicontinuous function $u$ satisfies $-\Delta_{\infty} u \leq 0$ on its domain in the viscosity sense if and only if for each $v(x)=$ $a|x-z| \in \mathcal{C}_{\infty}$ and bounded open set $U \subset \mathbb{R}^{n} \backslash\{z\}$ whose closure is a compact subset of the domain of $u$, one has

$$
u(x)-v(x) \leq \sup _{\partial U}(u-v) \text { for } x \in U .
$$

In this note we put this observation in a general context. Via this context we strip away the ad hoc appearance of the proof of this fact in [4] (itself an adaptation of a proof in Jensen [7). The proof, including Jensen's use of it to show that functions with a certain extremal property are infinity harmonic, is "automatic" when viewed this way. These are simple matters. However, our motivation was not to see the workings of this proof more clearly, but rather a desire to know how general the phenomenon is. Can the subsolutions of other (possibly) degenerate

Received by the editors August 17, 2001 and, in revised form, February 20, 2002.

2000 Mathematics Subject Classification. Primary 35J70, 35J05, 35B50.

(C)2002 American Mathematical Society 
elliptic equations be characterized by requiring (0.2) for an explicit minimal set of $v$ 's? In this regard, we note that "viscosity subsolutions" are basically defined via (0.2) where the $v$ 's range over all strict classical supersolutions of the equation. A self-contained explanation of this simple fact is contained in Section 1. It is a standard result of nonlinear potential theory (see the book 6 and Lindqvist [9]) that $p$-subharmonic functions in the variational sense are exactly those functions which enjoy the comparison property with respect to $p$-harmonic functions. We seek more explicit and minimal classes, typically built from radial functions in the case of equations which are rotation invariant.

We provide a positive answer in the case of the Laplacian, whence the title of this paper, and in fact offer two interesting specific classes in this case. We briefly describe one of these now. Let $\Phi(r)=-r^{2-n}$ if $2<n$ and $\Phi(r)=\ln (r)$ if $n=2$ be the usual radial solutions. Then $u$ satisfies $-\Delta u \leq 0$ iff 0.2 holds for all $v$ 's which are linear combinations with positive coefficients of $n$ functions of the form $x \mapsto \Phi(|x-z|)$ where $z \in \mathbb{R}^{n}$ and appropriate $U$. For the alternative class, we refer to Section 2. Roughly speaking, these classes are minimal with respect to this property. Since the Laplacian is an odd operator, $u$ is harmonic if and only if $u$ and $-u$ are subharmonic; so a characterization of subharmonic functions provides a characterization of harmonic functions. The other operators appearing in this paper are odd as well.

The infinity Laplacian and the Laplacian correspond to $p=\infty$ and $p=2$, respectively, when considering the $p$-Laplacian, $\Delta_{p} u=\operatorname{div}\left(|D u|^{p-2} D u\right)$ (the correspondence for the case $p=\infty$ is a bit subtle, see [2], [7] and [4]). One now naturally wants to know about the general case. Here we obtain first an interesting positive result - there are indeed families to consider. That is, we show that linear combinations of radial $p$-superharmonic functions - even allowing different singularities and exponents - with appropriately signed coefficients are again $p$-superharmonic, that is, they satisfy $-\Delta_{p} u \geq 0$. We find this quite surprising and regard it as one of our main results. However, the other news is that these families do not suffice to characterize $p$-subharmonic functions in the manner just described. The situation remains a bit mysterious. We have not found a minimal class of functions with this property. As we see it, the fact that there is no straightforward generalization of the results for the cases $p=2$ and $p=\infty$ to other $p$ adds interest to the results in these special cases.

This paper is self-contained. In the next section, we set up the general framework of the discussion. There are no really new results in this painless section; rather it sets up some common observations in a convenient explicit way. In Section 2, we establish the results mentioned above concerning infinity subharmonic functions and subharmonic functions in this framework. The analysis involved after the issues are properly understood is linear algebra. The case $p=\infty$ immediately yields to the general point of view. However, the classical linear case $p=2$ turns out to be rather subtle and takes some time to treat. In Section 3, we construct our family of $p$-superharmonic functions and then show that the corresponding theorem is false for this class and $p \neq 2, \infty$.

We thank J. Manfredi, P. Lindqvist and C. de Boor for helpful comments and references. 


\section{Preliminaries}

We begin by setting some notation.

(i) $V, U$ denote open subsets of $\mathbb{R}^{n}$. $V \subset \subset U$ means that the closure of $V, \bar{V}$, is a compact subset of $U$. $\partial V$ is the boundary of $V$.

(ii) $u, v, w$ will denote real-valued functions defined on open subsets of $\mathbb{R}^{n}$. Their domains will be denoted by $\operatorname{dom}(u)$, etc. The phrase "a function $u$ in $\mathbb{R}^{n}$ " will denote such a function $u$.

(iii) The inner product of $x, y \in \mathbb{R}^{n}$ is $\langle x, y\rangle$ and $|x|^{2}=\langle x, x\rangle$.

(iv) If $z \in \mathbb{R}^{n} \backslash\{0\}$, then $\hat{z}=z /|z|$ is the unit vector in the direction of $z$.

(v) $z \otimes z$ is the linear operator $x \mapsto\langle x, z\rangle z$.

(vi) The open ball of radius $r$ and center $x$ is $B_{r}(x)$.

(vii) USC (LSC) is the set of functions $u$ in $\mathbb{R}^{n}$ such that $u$ is upper-semicontinuous (respectively, lower-semicontinuous) on $\operatorname{dom}(u)$.

For the moment, $p$ denotes an element of $\mathbb{R}^{n}$ and is not associated with $\Delta_{p}$. We assume $F(x, p, X)$ is a degenerate elliptic operator in $\mathbb{R}^{n}$ in the sense of [5]. That is, $F(x, p, X) \in \mathbb{R}$ for all $x, p \in \mathbb{R}^{n}$ and real $n \times n$ symmetric matrices $X$, and $F$ depends continuously on its argument $(x, p, X)$ and is nonincreasing with respect to $X$. Then the equation $F\left(x, D u, D^{2} u\right)=0$ is a (possibly) degenerate elliptic equation in the sense of [5]. For example, $F(p, X)=-\operatorname{trace}(X)$ corresponds to $-\Delta$ and $F(p, X)=-$ trace $((p \otimes p) X)=-\langle X p, p\rangle$ corresponds to $-\Delta_{\infty}$. We do not write $F(x, r, p, X)$ so as to allow $F\left(x, u, D u, D^{2} u\right)$, but this is merely because the examples in this paper do not have a $u$ dependence and the less notation the better.

The operator $F$ determines various classes of functions. We are interested, in particular, in viscosity subsolutions and strict classical supersolutions. The class of viscosity subsolutions will be denoted by $\underline{\mathrm{V}}(F)$ :

$\underline{\mathrm{V}}(F)=\left\{u \in\right.$ USC such that $F\left(x, D u, D^{2} u\right) \leq 0$ in dom $(u)$ in the viscosity sense $\}$.

The relevant definition of $F\left(x, D u, D^{2} u\right) \leq 0$ ( $F \leq 0$ for short) is implicit below. The class of strict classical supersolutions of $F=0$ will be denoted by $\overline{\operatorname{sc}}(F)$. Forgive the notation- $s$ is for "strict" and $c$ is for "classical". Underscores correspond to "sub" and overscores to "super". That is,

$$
\begin{aligned}
& \overline{\operatorname{sc}}(F)=\left\{C^{2} \text { functions } v \text { in } \mathbb{R}^{n}\right. \text { such that } \\
& \left.\qquad F\left(x, D v(x), D^{2} v(x)\right)>0 \text { for } x \in \operatorname{dom}(v)\right\} .
\end{aligned}
$$

The very definition of a viscosity solution $u$ of $F \leq 0$ (equivalently, a viscosity subsolution of $F=0$ ) on $U \subset \mathbb{R}^{n}$ can be phrased this way: $u$ is an uppersemicontinuous function in $\mathbb{R}^{n}$ with $\operatorname{dom}(u)=U$ such that if $v \in \overline{\operatorname{sc}}(F)$, then $u-v$ does not have any local maximum points in $\operatorname{dom}(u-v)=\operatorname{dom}(u) \cap \operatorname{dom}(v)$. We denote the set of upper-semicontinuous functions in $\mathbb{R}^{n}$ that have no local maxima by NLM:

$\mathrm{NLM}=\{u \in \mathrm{USC}$ such that $u$ does not have any local maximum points $\}$.

A related class is the set of functions $u$ satisfying the "maximum principle" in the following sense: for every $V \subset \subset \operatorname{dom}(u)$,

$$
u(x) \leq \sup _{y \in \partial V} u(y) \text { for } x \in V .
$$


The set of upper-semicontinuous functions with this property is denoted by MP:

$$
\mathrm{MP}=\{u \in \mathrm{USC}: u \text { satisfies the maximum principle }\} .
$$

NLM and MP are related by

$$
\mathrm{NLM} \subset \mathrm{MP} .
$$

Indeed, if $u \in \mathrm{USC}$ and $u \notin \mathrm{MP}$, then there is a set $V \subset \subset \operatorname{dom}(u)$ and $\hat{x} \in V$ such that $u(\hat{x})>\max _{\partial V} u$. Then $u$ assumes its maximum over $\bar{V}$ at a point of $V$ and $u \notin \mathrm{NLM}$. Of course, the inclusion is strict (e.g, MP contains the constant functions).

As noted above, $\underline{\mathrm{V}}(F)$ consists of the functions $u \in \mathrm{USC}$ such that for every $v \in \overline{\operatorname{sc}}(F), u-v \in$ NLM. However, it is also true that $u \in \underline{\mathrm{V}}(F)$ iff for every $v \in \overline{\operatorname{sc}}(F)$ we have $u-v \in \mathrm{MP}$. The following proposition records these observations:

Proposition 1.1. Let $u$ be an upper-semicontinuous function in $\mathbb{R}^{n}$. Then the following conditions are equivalent:

(i) $u \in \underline{\mathrm{V}}(F)$.

(ii) If $v \in \overline{\mathrm{sc}}(F)$, then $u-v \in \mathrm{NLM}$.

(iii) If $v \in \overline{\mathrm{sc}}(F)$, then $u-v \in \mathrm{MP}$.

As remarked, the equivalence of (i) and (ii) is essentially by definition. The implication (ii) $\Longrightarrow$ (iii) follows from (1.1). We set aside the simple proof of (iii) $\Longrightarrow$ (ii) until a more general statement is formulated below.

If $\mathcal{C} \subset$ LSC, then $\mathcal{C}$ may be used in place of $\overline{\mathrm{sc}}(F)$ in (ii) and (iii) above to define new classes of functions. We call these " $\underline{V}(\mathcal{C})$ " for " $\mathcal{C}$ - viscosity subsolutions" and "S$(\mathcal{C})$ " for "C - subharmonic functions". More precisely,

$$
\underline{\mathrm{V}}(\mathcal{C})=\{u \in \mathrm{USC} \text { such that if } v \in \mathcal{C}, \text { then } u-v \in \mathrm{NLM}\}
$$

and

$$
\underline{\mathrm{S}}(\mathcal{C})=\{u \in \mathrm{USC} \text { such that if } v \in \mathcal{C} \text {, then } u-v \in \mathrm{MP}\} .
$$

We make no apology for the abuse of notation, $\underline{\mathrm{V}}(F)$ vs. $\underline{\mathrm{V}}(\mathcal{C})$, as context always saves the day here. With this notation, $\underline{\mathrm{V}}(\overline{\mathrm{sc}}(F))=\underline{\mathrm{V}}(F)$ is the set of viscosity solutions of $F \leq 0$ and the proposition above states that $\underline{\mathrm{V}}(\overline{\mathrm{sc}}(F))=\underline{\mathrm{S}}(\overline{\mathrm{sc}}(F))$.

The function class $\overline{\mathrm{sc}}(F)$ has a variety of properties. For convenience now and later, we introduce the following notation. If $v, w$ are functions in $\mathbb{R}^{n}$, then

$$
v \prec \underset{\hat{x}}{\prec} w
$$

means that $\hat{x} \in \operatorname{dom}(v) \cap \operatorname{dom}(w)$ and $\hat{x}$ is a strict minimum point for $w-v$; i.e., there is an $r_{0}>0$ such that for $0<r \leq r_{0}$, there is a $\delta(r)>0$ such that $w(x)-v(x)-\delta(r)>w(\hat{x})-v(\hat{x})$ for $x \in \partial B_{r}(\hat{x})$.

Remark 1.2. We note the following simple facts. The statement $v \prec_{\hat{x}} w$ is the same as $v+a \prec_{\hat{x}} w+b$ for any constants $a, b$. Moreover, if $v, w$ are continuous, then $v \prec_{\hat{x}} w$ implies that for sufficiently small $\varepsilon>0$, there is a neighborhood $N \subset \subset$ $\operatorname{dom}(w) \cap \operatorname{dom}(v)$ of $\hat{x}$ such that $v(x)=w(x)+v(\hat{x})-w(\hat{x})-\varepsilon$ on $\partial N$. Indeed, the function on the left exceeds the function on the right at $x=\hat{x}$, while the function on the right exceeds that on the left on $\partial B_{r}(\hat{x})$ as soon as $\varepsilon<\delta(r)$ per the definition. Take $N$ to be the component of $\{x: v(x)>w(x)+v(\hat{x})-w(\hat{x})-\varepsilon\}$ containing $\hat{x}$. 
Here is a significant property of $\overline{\operatorname{sc}}(F)$ for us:

$$
v \in \overline{\operatorname{sc}}(F), \hat{x} \in \operatorname{dom}(v) \Longrightarrow \exists w \in \overline{\operatorname{sc}}(F) \text { with } v \underset{\hat{x}}{\prec} w .
$$

One standard way to do this demonstrates that $w$ may be taken as a polynomial $q$ of degree 2. Let $\lambda>0$ and put

$$
w_{\lambda}(x)=v(\hat{x})+\langle D v(\hat{x}), x-\hat{x}\rangle+\frac{1}{2}\left\langle D^{2} v(\hat{x})(x-\hat{x}), x-\hat{x}\right\rangle+\lambda|x-\hat{x}|^{2} .
$$

Via Taylor's theorem, $w_{\lambda}(x) \geq v(x)+\lambda|x-\hat{x}|^{2} / 2$ for $x$ near $\hat{x}$. Since

$$
F\left(\hat{x}, D w_{0}(\hat{x}), D^{2} w_{0}(\hat{x})\right)=F\left(\hat{x}, D v(\hat{x}), D^{2} v(\hat{x})\right)>0,
$$

we also have $F\left(x, D w_{\lambda}(x), D^{2} w_{\lambda}(x)\right)>0$ for $x$ near $\hat{x}$ and $\lambda$ near 0 . Thus for $\lambda$ sufficiently small, $q=w_{\lambda}$ restricted to a suitable neighborhood of $\hat{x}$ has the desired properties.

The property (1.4 might be enjoyed by an arbitrary set of functions $\mathcal{C}$ in place of $\overline{\operatorname{sc}}(F)$. What we will use in the next result is a generalization of this condition to a pair $\mathcal{C}_{1}, \mathcal{C}_{2}$ of sets of functions. Here then is a proof of the unproven assertion of Proposition 1.1 in a generality needed in the next section.

Lemma 1.3. Let $\mathcal{C}_{1}, \mathcal{C}_{2} \subset$ LSC. Assume that if $v \in \mathcal{C}_{1}$ and $\hat{x} \in \operatorname{dom}(v)$, then there exists $w \in \mathcal{C}_{2}$ such that

$$
v \prec w .
$$

Then $\underline{\mathrm{S}}\left(\mathcal{C}_{2}\right) \subset \underline{\mathrm{V}}\left(\mathcal{C}_{1}\right)$.

Proof. Suppose $u \in \mathrm{USC}$ but $u \notin \underline{\mathrm{V}}\left(\mathcal{C}_{1}\right)$. Then there exists $v \in \mathcal{C}_{1}, \hat{x} \in \operatorname{dom}(v) \cap$ $\operatorname{dom}(u)$ for which $u(x)-v(x) \leq u(\hat{x})-v(\hat{x})$ for $x$ near $\hat{x}$. Let $w \in \mathcal{C}_{2}$ and $v \prec_{\hat{x}} w$ per the assumptions. Then for each small $r$ there is a $\delta(r)>0$ such that for $x \in \partial B_{r}(\hat{x})$,

$$
\begin{aligned}
u(x)-w(x) & =u(x)-v(x)+v(x)-w(x) \\
& \leq u(\hat{x})-v(\hat{x})+v(\hat{x})-w(\hat{x})-\delta(r) \\
& =u(\hat{x})-w(\hat{x})-\delta(r) .
\end{aligned}
$$

In particular, $u-w$ is not bounded above in $B_{r}(\hat{x})$ by its supremum on the boundary, whence $u \notin \underline{\mathrm{S}}\left(\mathcal{C}_{2}\right)$.

Proof of Proposition 1.1 (iii) $\Longrightarrow$ (ii). If $u$ satisfies (iii), then $u \in \underline{\mathrm{V}}(\overline{\mathrm{sc}}(F))$. Taking $\mathcal{C}_{1}=\mathcal{C}_{2}=\overline{\operatorname{sc}}(F)$ in Lemma [1.3] the hypotheses of the lemma are satisfied in view of the remarks above. Thus the lemma asserts that $u \in \underline{\mathrm{S}}(\overline{\operatorname{sc}}(F))$, which is (ii).

Let us outline, in the light of the above remarks, a sufficient program to show that $\underline{\mathrm{S}}(\mathcal{C})=\underline{\mathrm{V}}(F)$ when we are given a class $\mathcal{C}$ and an operator $F$. We know that if $v \in \underline{\mathrm{V}}(F)$ and $\hat{x} \in \operatorname{dom}(v)$, then there is a quadratic polynomial $q \in \overline{\operatorname{sc}}(F)$ (with a suitable domain) such that $v \prec_{\hat{x}} q$. If we can then find an element $w \in \mathcal{C}$ such that $q \prec_{\hat{x}} w$, we also have $v \prec_{\hat{x}} w$ and thus verify the conditions of Lemma 1.3 with $\mathcal{C}=\overline{\mathrm{sc}}(F)$. It then follows that $\underline{\mathrm{S}}(\mathcal{C}) \subset \underline{\mathrm{V}}(F)$. Next, we need to show that $\underline{\mathrm{V}}(F) \subset \underline{\mathrm{S}}(\mathcal{C})$. In our examples, it will be the case that $\mathcal{C}$ consists of classical solutions of $F \geq 0$. In this case, $\underline{\mathrm{V}}(F) \subset \underline{\mathrm{S}}(\mathcal{C})$ amounts to the comparison property between the classical supersolutions in $\mathcal{C}$ and viscosity solutions of $F \leq 0$. This is a result which must be established case by case. We formulate these remarks as a proposition. 
Proposition 1.4. Let $\mathcal{C}$ be a collection of functions in $\mathbb{R}^{n}$. Then $\underline{\mathrm{S}}(\mathcal{C})=\underline{\mathrm{V}}(F)$ provided that

$$
\underline{\mathrm{V}}(F) \subset \underline{\mathrm{S}}(\mathcal{C})
$$

and for each quadratic polynomial $q$ solving $F\left(x, D q(x), D^{2} q(x)\right)>0$ on an open set $\operatorname{dom}(q) \subset \mathbb{R}^{n}$ and $\hat{x} \in \operatorname{dom}(q)$,

$$
\text { there exists } w \in \mathcal{C} \text { such that } q \underset{\hat{x}}{\prec} w \text {. }
$$

Remark 1.5. The task of verifying (1.7) may be simplified in practice by determining normal forms for $q$. For example, in this regard one might encounter the following groups of order-preserving (and $\prec$ preserving) transformations $T$ on functions: $T(v)(x)=v\left(x+x_{0}\right)$ (translations), $T(v)(x)=v(B x)$ where $B$ is a unitary matrix (unitary transformations), $T(v)(x)=A(x)+v(x)$ where $A$ is an affine function (addition of affine functions), $T(v)(x)=v(\mu x)$ where $\mu>0$ (scaling). In the case of translations, $q \prec_{\hat{x}} w$ is converted into $T(q) \prec_{\hat{x}-x_{0}} T(w)$, etc. When $\overline{\operatorname{sc}}(F)$ and $\mathcal{C}$ are invariant under translations, we can simply study the problem $q \prec_{0} w$. When they are invariant under unitary transformations, we can assume $D^{2} q$ is diagonal, and so on.

Remark 1.6. Elementary calculus tells us that if $v, w \in C^{2}$ and $D w(\hat{x})=D v(\hat{x})$, $D^{2} w(\hat{x})>D^{2} v(\hat{x})$, then $v \prec_{\hat{x}} w$ holds. Let us also note, for future use, another consequence of elementary calculus. Let $\lambda_{\max }(X)$ be the maximum eigenvalue of the symmetric matrix $X$. Let $u$ be a $C^{2}$ function in $\mathbb{R}^{n}$. If $\lambda_{\max }\left(D^{2} u(x)\right)>0$ whenever $x \in \operatorname{dom}(u)$ and $D u(x)=0$, then $u \in \mathrm{MP}$. This is because, under this condition for each $x \in \operatorname{dom}(u)$ and $r>0$, there is a $y \in B_{r}(x) \cap \operatorname{dom}(u)$ such that $u(y)>u(x)$.

\section{Subharmonic AND $\infty$-Subharmonic}

As a first illustration, we revisit the case of $-\Delta_{\infty}$. For the convenience of browsers, we spell out the fact proved without using our notation.

Theorem 2.1. Let $u \in \mathrm{USC}$. Then $-\Delta_{\infty} u \leq 0$ in $\operatorname{dom}(u)$ if and only if for each $a \geq 0, z \in \mathbb{R}^{n}$, and $V \subset \subset \operatorname{dom}(u) \cap\left(\mathbb{R}^{n} \backslash\{z\}\right)$,

$$
u(x)-a|x-z| \leq \max _{y \in \partial V}(u(y)-a|y-z|) \text { for } x \in V .
$$

Proof. The instance $\underline{\mathrm{V}}\left(-\Delta_{\infty}\right) \subset \underline{\mathrm{S}}\left(\mathcal{C}_{\infty}\right)$ (recall (0.1)) of (1.6) holds here because $\mathcal{C}_{\infty}$ consists of classical solutions of $-\Delta_{\infty} u=0$ which perturb to strict classical supersolutions (see [4] and Jensen [7]). The class ([0.1) is invariant under translations, as is $\overline{\operatorname{sc}}\left(-\Delta_{\infty}\right)$. Given a quadratic polynomial $q \in \overline{\operatorname{sc}}\left(-\Delta_{\infty}\right)$ for which we seek to exhibit $w \in \mathcal{C}_{\infty}$ for which $q \prec \hat{x} w$, we may therefore assume that $\hat{x}=0$. The relation $-\Delta_{\infty} q(0)>0$ then amounts to

$$
-\langle S y, y\rangle>0 \text { where } S=D^{2} q(0), y=D q(0) .
$$

Assuming (2.2), we seek $w(x)=a|x-z| \in \mathcal{C}_{\infty}$ such that

$$
\text { (i) } D w(0)=y \text { and (ii) } D^{2} w(0)>S \text {. }
$$

Putting $\hat{z}=z /|z|$ we have

$$
D w(0)=-a \hat{z}, \quad D^{2} w(0)=\frac{a}{|z|}(I-\hat{z} \otimes \hat{z}) .
$$


Thus (2.3) amounts to

$$
\text { (i) }-a \hat{z}=y \text { and (ii) } \frac{a}{|z|}(I-\hat{z} \otimes \hat{z})>S
$$

or

$$
\text { (i) }-a \hat{z}=y \text { and (ii) } I>\hat{z} \otimes \hat{z}+\mu S \text { where } \mu=\frac{|z|}{a} \text {. }
$$

From (i), $a=|y|=|D q(0)|$ and $\hat{z}=-\hat{y}=-\widehat{D q(0)}$. Thus, if we can find $\mu>0$ such that $I>\hat{y} \otimes \hat{y}+\mu S$ holds, then putting $\hat{z}=-\hat{y},|z|=\mu|y|$ completes the construction. Clearly there is such a $\mu>0$ only if $\langle S \hat{y}, \hat{y}\rangle<0$; this is satisfied by (2.2). This condition is also sufficient. Indeed, writing $x \in \mathbb{R}^{n}$ as $x=\alpha \hat{y}+y^{\perp}$ where $\left\langle\hat{y}, y^{\perp}\right\rangle=0$ for $\epsilon>0$ we have

$$
\begin{aligned}
\langle(\hat{y} \otimes \hat{y}+\mu S) x, x\rangle & =\alpha^{2}(1+\mu\langle S \hat{y}, \hat{y}\rangle)+\mu\left(2 \alpha\left\langle S \hat{y}, y^{\perp}\right\rangle+\left\langle S y^{\perp}, y^{\perp}\right\rangle\right) \\
& \leq \alpha^{2}\left(1+\mu\left(\langle S \hat{y}, \hat{y}\rangle+\epsilon|S \hat{y}|^{2}\right)\right)+\mu\left(\frac{1}{\varepsilon}+\|S\|\right)\left|y^{\perp}\right|^{2} .
\end{aligned}
$$

Now choose $\varepsilon>0$ so small that $\langle S \hat{y}, \hat{y}\rangle+\epsilon|S \hat{y}|^{2}<0$ and then $\mu>0$ so small that $\mu(1 / \varepsilon+\|S\|)<1$. It is evident that then $I>\hat{y} \otimes \hat{y}+\mu S$.

Remark 2.2. Suppose that $u$ does not solve $-\Delta_{\infty} u=0$. Passing to $-u$ if necessary and using translations, we may assume that $-u$ fails to be a subsolution at $x=0$, that is, there exists a quadratic $q \in \overline{\mathrm{sc}}\left(-\Delta_{\infty}\right)$ with $\left\langle D^{2} q(0) D q(0), D q(0)\right\rangle<0$ for which 0 is a strict maximum point of $u-q$. Then for small $\lambda>0$,

$$
\begin{aligned}
u(-\lambda D q(0))-u(0) & <q(-\lambda D q(0))-q(0) \\
& =-\lambda\langle D q(0), D q(0)\rangle+\frac{\lambda^{2}}{2}\left\langle D^{2} q(0) D q(0), D q(0)\right\rangle \\
& <-\lambda\langle D q(0), D q(0)\rangle=-\lambda|D q(0)||D q(0)| .
\end{aligned}
$$

Hence $|D q(0)|$ is NOT a Lipschitz constant for $u$ in any neighborhood of 0 . However, we have shown above that there is a $w(x)=|D q(0)||x-z|$ such that $q \prec_{0} w$. In view of Remark 1.2. this guarantees that $u$ agrees with a function ( $w$ plus a constant) with Lipschitz constant $|D q(0)|$ on the boundary of an open neighborhood of 0 . The point here is summarized by saying that if $u$ is not a solution of $-\Delta_{\infty} u=0$, then it is not "absolutely minimizing Lipschitz", as originally proved by Jensen [7. Here we see how natural the proof can be.

Remark 2.3. The fact that absolute minimizers for more general functionals than $|D u|^{2}$ satisfy corresponding degenerate elliptic pdes has recently been proved in great generality; see Barron, Jensen and Wang [1] and Crandall [3]. In particular, the efficient proof of [3] was illuminated by the current work and also implies generalizations of the example just treated. That is, solutions of certain degenerate elliptic equations $F\left(x, u, D u, D^{2} u\right)=0$ are determined as in this work via classes of solutions of corresponding first-order pdes $f(x, u, D u)=$ constant; note that $w=a|x-z|$ is a solution of $|D w|^{2}=a^{2}$.

We turn to the case of the Laplacian. Put

$$
\Phi(r)= \begin{cases}-\frac{1}{(n-2) r^{n-2}} & \text { if } n>2 \\ \ln (r) & \text { if } n=2 .\end{cases}
$$


Let

$$
\mathcal{C}_{2}=\left\{x \mapsto \sum_{j=1}^{n} a_{j} \Phi\left(\left|x-z^{j}\right|\right): a_{j} \in \mathbb{R}^{+}, z^{j} \in \mathbb{R}^{n} \text { for } 1 \leq j \leq n\right\} .
$$

The subscript 2 corresponds to the Laplacian being the case $p=2$ of the $p$ Laplacian. Note that $a_{j} \in \mathbb{R}^{+}$in (2.6); i.e., the coefficients are nonnegative. We understand that the domains of the functions in $\mathcal{C}_{2}$ are to be $\mathbb{R}^{n}$ with the singularities deleted. We will prove that $\underline{\mathrm{V}}(-\Delta)=\underline{\mathrm{S}}\left(\mathcal{C}_{2}\right)$.

A less elegant looking family of functions also has the desired property. We put

$$
\hat{\mathcal{C}}_{2}=\left\{x \mapsto\langle a, x\rangle+\sum_{j=1}^{n-1} \Phi\left(\left|x-z^{j}\right|\right): a, z^{1}, \ldots, z^{n-1} \in \mathbb{R}^{n}\right\} .
$$

We emphasize that there are $n$ multiples of the fundamental solution in the definition of $\mathcal{C}_{2}$ and $n-1$ such terms all with the coefficient 1 in the definition of $\hat{\mathcal{C}}_{2}$. These numbers $n$ and $n-1$ are minimal for the validity of the next result, which we again state without invoking notation.

Theorem 2.4. Let $u \in$ USC. Then the following are equivalent:

(i) $-\Delta u \leq 0$ in $\operatorname{dom}(u)$.

(ii) For all $0<a_{j}, z^{j} \in \mathbb{R}^{n}, j=1, \ldots, n$ and $V \subset \subset \operatorname{dom}(u) \backslash\left\{z^{1}, \ldots, z^{n}\right\}$,

$$
u(x)-\sum_{j=1}^{n} a_{j} \Phi\left(\left|x-z^{j}\right|\right) \leq \max _{y \in \partial V}\left(u(y)-\sum_{j=1}^{n} a_{j} \Phi\left(\left|y-z^{j}\right|\right)\right) \text { for } x \in V .
$$

(iii) For all $a \in \mathbb{R}^{n}, z^{1}, \ldots, z^{n-1} \in \mathbb{R}^{n}$, and $V \subset \subset \operatorname{dom}(u) \backslash\left\{z^{1}, \ldots, z^{n-1}\right\}$,

$$
u(x)-\left(\langle a, x\rangle+\sum_{j=1}^{n-1} \Phi\left(\left|x-z^{j}\right|\right)\right) \leq \max _{y \in \partial V}\left(u(y)-\left(\langle a, y\rangle+\sum_{j=1}^{n-1} \Phi\left(\left|y-z^{j}\right|\right)\right)\right)
$$

for $x \in V$.

Before turning to the proof of Theorem 2.4 we further set the stage. At this point, the reader may skip ahead to the proof of the theorem below without loss of continuity. Returning to Theorem 2.1, observe that if $u$ is a solution of $-\Delta_{\infty} u \leq 0$, then (2.1) holds with arbitrary $a \in \mathbb{R}$, but that it suffices for (2.1) to hold with $a>0$ to guarantee that $-\Delta_{\infty} u \leq 0$. In Theorem 2.4 (ii), the coefficients are also signed, that is, $a_{j}>0$, while in (iii) they are unity. Later we will see why the classes $\mathcal{C}_{2}$ and $\hat{\mathcal{C}}_{2}$ are in some sense minimal in this signed formulation. What we want to do now is provide a glimpse of why all the terms above are needed by exhibiting a simple explicit quadratic function $u$ in $\mathbb{R}^{3}$ such that $x \mapsto \pm u(x)-a \Phi(|x-z|) \in \mathrm{MP}$ for every $z \in \mathbb{R}^{3}, a \in \mathbb{R}$, but $u$ is NOT harmonic. This establishes that the comparison property with respect to all multiples of all translates of the fundamental solution does not characterize harmonicity. According to (ii) above, comparison in this sense with respect to linear combinations of $n$ such functions does, and we may use appropriately signed coefficients.

Let $u$ be the homogeneous quadratic polynomial satisfying

$$
D^{2} u \equiv\left(\begin{array}{ccc}
-4 & 0 & 0 \\
0 & 0 & 0 \\
0 & 0 & 3
\end{array}\right)=: S
$$


Since trace $(S)=-1<0, u$ is strictly superharmonic. It follows that $-u-a \Phi(\mid x-$ $z \mid) \in \mathrm{MP}$ for all $a, z$. The interesting fact is that $u-a \Phi(|x-z|) \in \operatorname{MP}$ for all $a, z$. Let us first note that

$$
D \Phi(|x|)=\frac{1}{|x|^{3}} x, \quad D^{2} \Phi(|x|)=\frac{1}{|x|^{3}}(I-3 \hat{x} \otimes \hat{x}) .
$$

According to Remark 1.6, if we show that $\lambda_{\max }\left(D^{2} u-a D^{2} \Phi(|x-z|)\right)>0$ no matter the choices of $a, z, x$, we are done. That is, relabeling $x-z$ to $x$ again, we claim that

$$
X=\left(\begin{array}{ccc}
-4 & 0 & 0 \\
0 & 0 & 0 \\
0 & 0 & 3
\end{array}\right)+\frac{a}{|x|^{3}}(-I+3 \hat{x} \otimes \hat{x})
$$

always has a positive eigenvalue. In the case $a>0$, this is relatively simple. Putting $R=a /|x|^{3}$, the second summand has an eigenvalue $2 R$ and for all eigenvalues to be nonpositive, this has to be "canceled out" by the least eigenvalue -4 of the first summand, that is, $-4+2 R \leq 0$ or $R \leq 2$. However, then the eigenvalue 3 of the first summand cannot be "canceled out" by the least eigenvalue $-R$ of the second summand, whence there is a positive eigenvalue. For the case in which $a$ is negative, we put $R=-a /|x|^{3} \geq 0$. Computing $\langle X y, y\rangle$ subject to $\langle\hat{x}, y\rangle=0$ leads to

$$
\langle X y, y\rangle=(-4+R) y_{1}^{2}+R y_{2}^{2}+(3+R) y_{3}^{2} .
$$

For this to be nonpositive for all $y \perp \hat{x}$ obviously requires $4>R$ and

$$
y_{1}^{2} \geq \frac{R}{4-R} y_{2}^{2}+\frac{3+R}{4-R} y_{3}^{2} \text { for } y \perp x,
$$

but it is obvious that the solid cone described by the inequality contains no planes through the origin. Hence again $X$ has a positive eigenvalue. Alternatively, this follows from the min-max characterization of eigenvalues and the fact that $X+$ $3 R \hat{x} \otimes \hat{x}$ has at least two positive eigenvalues. We are done: $\pm u-a \Phi(|x-z|) \in \mathrm{MP}$ for all $a, z$ but $u$ is not harmonic. Hereafter, all our coefficients will be of fixed sign.

We return to the proof of Theorem 2.4 Now for either $\mathcal{C}=\mathcal{C}_{2}$ or $\mathcal{C}=\hat{\mathcal{C}}_{2}, \mathcal{C}$ consists of classical solutions of $-\Delta u=0$ that perturb to strict classical supersolutions and therefore $\underline{\mathrm{V}}(-\Delta) \subset \underline{\mathrm{S}}(\mathcal{C})$. The rest of what we must show, in view of translation invariance, is that if $q$ is a quadratic polynomial and $-\Delta q>0$, then there is a $w \in \mathcal{C}_{2}$ (respectively, $w \in \hat{\mathcal{C}}_{2}$ ) such that $q \prec_{0} w$.

We begin with the simpler proof, the case of $\hat{\mathcal{C}}_{2}$. Elements of this proof are also required in the more demanding case of $\mathcal{C}_{2}$.

Proof of Theorem 2.4 (iii) $\Longrightarrow$ (i). Let $v=q$ be a quadratic polynomial in $\overline{\operatorname{sc}}(-\Delta)$. Via invariance under translations and addition of affine functions, we may assume that $q$ is a homogeneous polynomial of degree 2 , and, since $-\Delta q>0$, trace $\left(D^{2} q\right)<$ 0 . Moreover, via invariance under addition of affine functions, which also leaves second derivatives invariant, all we have to do is to show that if $S=-D^{2} q$ (hence $\operatorname{trace}(S)>0$ ), we can find $z^{j} \in \mathbb{R}^{n} \backslash\{0\}$ such that

$$
w(x)=\sum_{j=1}^{n-1} \Phi\left(\left|x-z^{j}\right|\right)
$$


satisfies

$$
D^{2} w(0)>-S .
$$

The condition $D w(0)=D q(0)=0$ is then obtained via adding a linear function.

If $w$ is given by (2.9) and $\Phi$ by (2.5), a computation shows that

$$
D^{2} w(0)=\sum_{j=1}^{n-1} \frac{1}{\left|z^{j}\right|^{n}}\left(I-n\left(\hat{z}^{j} \otimes \hat{z}^{j}\right)\right) .
$$

With a little arithmetic, the problem (2.10) transforms:

$$
\sum_{j=1}^{n-1} \frac{1}{\left|z^{j}\right|^{n}}\left(I-n\left(\hat{z}^{j} \otimes \hat{z}^{j}\right)\right)>-S \Longleftrightarrow I+r S>n \sum_{j=1}^{n-1} \alpha_{j} \hat{z}^{j} \otimes \hat{z}^{j}
$$

where

$$
r=\left(\sum_{j=1}^{n-1} \frac{1}{\left|z^{j}\right|^{n}}\right)^{-1}>0 \text { and } \alpha_{j}=\frac{r}{\left|z^{j}\right|^{n}}
$$

and we notice that

$$
0<\alpha_{j} \text { and } \sum_{j=1}^{n-1} \alpha_{j}=1
$$

The linear algebra problem we then seek to solve is this: given a symmetric $n \times n$ matrix $S$ with trace $S>0$, find a number $r>0$, unit vectors $\hat{z}^{j} \in \mathbb{R}^{n}$ and $\alpha_{j}>0$ satisfying (2.13) such that

$$
I+r S>n \sum_{j=1}^{n-1} \alpha_{j} \hat{z}^{j} \otimes \hat{z}^{j}
$$

Given $r, \alpha_{j}$ satisfying these conditions, one then easily constructs solutions of the left-hand side of (2.11) via (2.12).

For future use, let us also note that if we seek $w$ as in (2.9) but with a general number $l, 1 \leq l \leq n$ of singularities, say

$$
w(x)=\sum_{j=1}^{l} \Phi\left(\left|x-z^{j}\right|\right),
$$

the same analysis leads to the problem

$$
\begin{gathered}
I+r S>n \sum_{j=1}^{l} \alpha_{j} \hat{z}^{j} \otimes \hat{z}^{j}, \\
r=\left(\sum_{j=1}^{l} \frac{1}{\left|z^{j}\right|^{n}}\right)^{-1} \text { and } \alpha_{j}=\frac{r}{\left|z^{j}\right|^{n}} ;
\end{gathered}
$$

so we have now

$$
0<\alpha_{j} \text { and } \sum_{j=1}^{l} \alpha_{j}=1 .
$$

Later we will see that we must take $l \geq n-1$ for some choices of $S$. 
The solvability of (2.14) is easily exhibited: let $\left\{\hat{z}^{j}\right\}$ be an orthonormal basis of eigenvectors of $S$ and hence of $I+r S$. Choose $r>0$ but sufficiently small so that $I+r S>0$. Let $\left\{\lambda_{j}\right\}_{j=1}^{n}$ be the eigenvalues of $S$ repeated according to their multiplicity. Order things so that

$$
\lambda_{n} \leq \lambda_{n-1} \leq \ldots \leq \lambda_{1} .
$$

Then trace $(S)>0$ guarantees that

$$
T:=\lambda_{1}+\lambda_{2}+\ldots+\lambda_{n-1}>0 .
$$

This is because trace $(S)=\lambda_{1}+\lambda_{2}+\ldots+\lambda_{n-1}+\lambda_{n}>0$ and either $\lambda_{n} \geq 0$, in which case (2.19) holds due to (2.18), or $\lambda_{n}<0$, in which case (2.18) holds because $T$ exceeds trace $(S)$.

Now we may choose $r>0$ so that

$$
1+r \lambda_{n}>0 \text { and } n-1+r T>n .
$$

Indeed, if $\lambda_{n} \geq 0$, we need only take $r$ sufficiently large. On the other hand, if $\lambda_{n}<0$, then (2.20) requires that

$$
\frac{1}{T}<r<\frac{1}{-\lambda_{n}}
$$

which can be achieved provided that $-\lambda_{n}<T$ or $\lambda_{1}+\cdots+\lambda_{n}>0$, which is the condition trace $(S)>0$. Hence we can choose $r>0$ so that (2.20) holds; then

$$
\begin{aligned}
I+r S & =\sum_{j=1}^{n}\left(1+r \lambda_{j}\right) \hat{z}^{j} \otimes \hat{z}^{j}=\sum_{j=1}^{n-1}\left(1+r \lambda_{j}\right) \hat{z}^{j} \otimes \hat{z}^{j}+\left(1+r \lambda_{n}\right) \hat{z}^{n} \otimes \hat{z}^{n} \\
& =\frac{n-1+r T}{n} n\left(\sum_{j=1}^{n-1} \frac{1+r \lambda_{j}}{n-1+r T} \hat{z}^{j} \otimes \hat{z}^{j}+\frac{1+r \lambda_{n}}{n-1+r T} \hat{z}^{n} \otimes \hat{z}^{n}\right) \\
& >n\left(\sum_{j=1}^{n-1} \frac{1+r \lambda_{j}}{n-1+r T} \hat{z}^{j} \otimes \hat{z}^{j}+\frac{1+r \lambda_{n}}{n-1+r T} \hat{z}^{n} \otimes \hat{z}^{n}\right) \\
& \geq n \sum_{j=1}^{n-1} \frac{1+r \lambda_{j}}{n-1+r T} \hat{z}^{j} \otimes \hat{z}^{j}
\end{aligned}
$$

where $T$ is given by (2.19). The strict inequality above is due to (2.20) and the fact that the matrix following the strict inequality is strictly positive. Hence we have solved (2.14) with $\alpha_{j}=\left(1+r \lambda_{j}\right) /(n-1+r T)$.

Remark 2.5. The construction above showed that, in the definition of the class $\hat{\mathcal{C}}_{2}$, we could have required the $z^{j}$ to be pairwise orthogonal. The result still holds with the resulting smaller class. Moreover, it is necessary to take at least " $n-1$ singularities" in the definition of $\hat{\mathcal{C}}_{2}$ as we now show. If $1 \leq l<n-1$, then there are choices of $S$ for which (2.15) has no solutions. This fact is implicit in the construction above. We have

$$
\operatorname{trace}\left(n \sum_{j=1}^{l} \alpha_{j} \hat{z}^{j} \otimes \hat{z}^{j}\right)=n \sum_{j=1}^{l} \alpha_{j}=n ;
$$

so the maximal eigenvalue of the right-hand side of (2.15) (which has rank at most $l$ ) is at least $n / l$. Then any solution of (2.15) requires $I+r S$ to have an eigenvalue 
of at least $n / l$ while all eigenvalues are positive. If $S$ is the diagonal matrix with $n-1$ eigenvalues of 1 and $-n+1+\gamma$ with $0<\gamma<n-1$ as its remaining eigenvalue, then $r$ must satisfy

$$
1+r>\frac{n}{l} \text { and } 1+r(-n+1+\gamma)>0
$$

which requires

$$
l>\frac{n(n-1-\gamma)}{n-\gamma} .
$$

Thus we may take $\gamma$ sufficiently small to require $l>n-2$.

Proof of Theorem 2.4(ii) $\Longrightarrow$ (i). We turn to the proof for the class $\mathcal{C}_{2}$. Let $q$ be a quadratic polynomial with trace $\left(D^{2} q\right)<0$ and seek $w \in \mathcal{C}_{2}$ in the form

$$
w=\sum_{j=1}^{n} a_{j}\left|z^{j}\right|^{n-1} \Phi\left(\left|x-z^{j}\right|\right)
$$

where the $a_{j}>0$ and $z^{j}$ are such that

$$
D w(0)=b:=D q(0), D^{2} w(0)>D^{2} q .
$$

The normalization in the coefficients of $w$ is for later convenience. Doing the computations, these conditions amount to

$$
-\sum_{i=1}^{n} a_{i} \hat{z}^{i}=b \text { and } \sum_{i=1}^{n} \frac{a_{i}}{\left|z^{i}\right|}\left(I-n\left(\hat{z}^{i} \otimes \hat{z}^{i}\right)\right)>-S
$$

where $S=-D^{2} q$ is a matrix with positive trace.

Letting $\left\{\hat{z}^{j}\right\}$ be an orthonormal basis of eigenvectors of $S$, the first condition of (2.22) determines the $a_{i}$. Replacing $\hat{z}_{i}$ by $-\hat{z}_{i}$ if necessary, we can assume that $a_{i} \geq 0$. There are two cases. Either all the $a_{i}$ are positive or some are 0 . Remark 2.5] implies that we cannot always solve the second relation of (2.22) when more than 1 of the $a_{i}$ are zero, and in fact we cannot solve (2.22) in general with the ansatz that the $\hat{z}^{i}$ are an orthonormal basis of eigenvectors of $S$. However, this ansatz does suffice when all the coordinates of $b$ in a basis of eigenvectors of $S$ are nonzero. This follows from the line of argument above. Let $a_{i}>0$ for $i=1, \cdots, n$ be such that the first relation of (2.22) holds and then seek positive numbers $\left|z^{i}\right|$ such that the second relation holds. Relabeling $\beta_{i}=a_{i} /\left|z^{i}\right|$, the $\beta_{i}$ are arbitrary positive numbers and the second relation of (2.22) rewrites to

$$
I+\frac{1}{\sum_{j=1}^{n} \beta_{j}} S>n \sum_{i=1}^{n} \frac{\beta_{i}}{\sum_{j=1}^{n} \beta_{j}} \hat{z}^{i} \otimes \hat{z}^{i} .
$$

Similar, but simpler, manipulations to those leading to (2.21) show that

$$
I+r S>n \sum_{i=1}^{n} \alpha_{i} \hat{z}^{i} \otimes \hat{z}^{i}
$$

where the $\alpha_{i}=\left(1+r \lambda_{i}\right) /(n+r \operatorname{trace}(S))$ are positive and sum to 1 as soon as $r>0$ is sufficiently small to guarantee that $I+r S>0$. Then

$$
\beta_{i}=\frac{\alpha_{i}}{r}
$$

provides our solution. 
We turn to the case where there is no basis of eigenvectors of $S$ in which $b$ has only positive coordinates. The extreme case is $b=0$. We treat this by recalling from the proof of (iii) implies (i), that there are positive numbers $r, \kappa_{j}$ and eigenvectors $z^{j}$ of $S$ such that

$$
I+r S>n \sum_{j=1}^{n-1} \kappa_{j} \hat{z}^{j} \otimes \hat{z}^{j} \text { and } \sum_{j=1}^{n-1} \kappa_{j}=1 .
$$

Now we take these same $z^{j}$ for $j=1, \ldots, n-1$ and then use (2.22) with $a_{j}=1$ for $j=1,2, \ldots, n-1$ to determine $z^{n}$ :

$$
z^{n}:=-\left(z^{1}+\cdots+z^{n-1}\right) \text { and so } z^{1}+\cdots+z^{n-1}+z^{n}=b=0 .
$$

Since the inequality (2.24) is strict, for small $\mu>0$ we have

$$
I+r S>n\left(\sum_{j=1}^{n-1}\left(\kappa_{j}-\mu\right) \hat{z}^{j} \otimes \hat{z}^{j}+(n-1) \mu \hat{z}^{n} \otimes \hat{z}^{n}\right) .
$$

The coefficients of the $\hat{z}^{j} \otimes \hat{z}^{j}$ are positive and sum to one if $\mu$ is sufficiently small.

The remaining case is the case $b \neq 0$, but some components of $b$ in every eigenbasis for $S$ are zero. We resolve this by perturbing to the case already solved. Let $S$ be a symmetric matrix with

$$
\operatorname{trace}(S)>0
$$

and $b \in \mathbb{R}^{n} \backslash\{0\}$. We claim that then there is a symmetric matrix $S^{\prime}$ such that

$$
S^{\prime} \text { has simple eigenvalues, } S \geq S^{\prime}, \operatorname{trace}\left(S^{\prime}\right)>0
$$

and

$$
\text { if } z \text { is a nonzero eigenvector of } S^{\prime} \text {, then }\langle b, z\rangle \neq 0 \text {. }
$$

There are many ways to produce an $S^{\prime}$ as desired. Here is one. Notice first that we may assume that the eigenvalues of $S$ are simple by diagonalizing $S$ and then judiciously decreasing multiple eigenvalues to split them up. We do this, preserving (2.25). Now assume that $\left\{z^{j}\right\}_{j=1}^{n}$ is an orthonormal basis of eigenvectors of $S$ with corresponding eigenvalues $\lambda_{j}$ :

$$
S z^{j}=\lambda_{j} z^{j} \text { for } j=1, \cdots, n \text {. }
$$

Assuming that $b$ is orthogonal to one or more of the $z^{j}$, we order things so that

$$
\left\langle b, z^{j}\right\rangle=0 \text { for } j=1, \ldots, l \text { and }\left\langle b, z^{j}\right\rangle \neq 0 \text { for } j=l+1, \ldots, n .
$$

Now suppose that $B$ is symmetric and consider what happens to the first eigenvector $z^{1}$ under the perturbation of $S$ by $-t B$ where $t$ is small. Let $Z(t)$ be the continuation of $z^{1}$ for small $t>0$ : that is, let $Z(t), \Lambda(t)$ be the unique unit vector and scalar that are continuous in small $t$ and satisfy:

$$
(S-t B) Z(t)=\Lambda(t) Z(t) \text { and } Z(0)=z^{1}, \Lambda(0)=\lambda_{1} .
$$

These functions exist and are continuously differentiable by standard perturbation theory. The arguments below remind the reader how this goes. Differentiating (2.30) and setting $t=0$ yields

$$
S Z^{\prime}(0)-\lambda_{1} Z^{\prime}(0)=\Lambda^{\prime}(0) z^{1}+B z^{1} .
$$

According to the Fredholm alternative, this equation is solvable iff $\Lambda^{\prime}(0) z^{1}+B z^{1}$ is orthogonal to $z^{1}$, which determines $\Lambda^{\prime}(0)$. Next, the condition that $Z(t)$ be a unit 
vector requires that $Z^{\prime}(0)$ be orthogonal to $z^{1}$ and, subject to this condition, (2.31) has a unique solution $\Lambda^{\prime}(0), Z^{\prime}(0)$ (thus essentially verifying the hypotheses of the implicit function theorem):

$$
\Lambda^{\prime}(0)=-\left\langle B z^{1}, z^{1}\right\rangle, \quad Z^{\prime}(0)=\sum_{j=2}^{n}\left(\lambda_{j}-\lambda_{1}\right)^{-1}\left\langle B z^{1}, z^{j}\right\rangle z^{j} .
$$

Thus

$$
\left.\frac{d}{d t}\langle b, Z(t)\rangle\right|_{t=0}=\sum_{j=2}^{n}\left(\lambda_{j}-\lambda_{1}\right)^{-1}\left\langle B z^{1}, z^{j}\right\rangle\left\langle b, z^{j}\right\rangle .
$$

We want (2.33) to be positive and construct a symmetric $B$ with this property, as well as $B \geq 0$. We begin the definition of $B$ by putting

$$
B z^{1}=z^{1}+\kappa z^{l+1} \text { where }\left(\lambda_{l+1}-\lambda_{1}\right)^{-1} \kappa\left\langle b, z^{l+1}\right\rangle=1
$$

so that the right-hand side of (2.33) is 1 . For the other basis vectors, we put

$$
B z^{l+1}=\kappa z^{1}+\mu z^{l+1} \text { and } B z^{j}=\mu z^{j} \text { for } j \in\{1,2, \ldots, n\}, j \neq 1, l+1 .
$$

Clearly $B$ is symmetric. If we choose $\mu$ sufficiently large, then $B$ is also positive because

$$
\left\langle B \sum_{j=1}^{n} a_{j} z^{j}, \sum_{j=1}^{n} a_{j} z^{j}\right\rangle=a_{1}^{2}+\mu \sum_{j=2}^{n} a_{j}^{2}+2 \kappa a_{1} a_{l+1} .
$$

Now we are done. For sufficiently small $t>0, S>S^{\prime}:=S-t B, S^{\prime}$ has positive trace, and $b$ is orthogonal to at most $l-1$ of the eigenvectors of $S^{\prime}$. Indeed, it is not orthogonal to the continuation of $z^{1}$ by construction (2.33) and (2.34) and it is not orthogonal to the continuation of $z^{j}$ for $l+1 \leq j \leq n$ for small $t$ by continuity. Repeating the process, if necessary, we can again reduce the number of orthogonalities by at least 1 , and so on until we are done. Of course, one can write down a $B$ that eliminates them all in one step, but that would come at the expense of writing more complicated formulas.

\section{3. $p$-SUPERHARMONIC FunCtions}

In this section, we consider the $p$-Laplacian, which is given on smooth functions by

$$
\Delta_{p} u=\operatorname{div}\left(|D u|^{p-2} D u\right)=|D u|^{p-4}\left(|D u|^{2} \Delta u+(p-2) \Delta_{\infty} u\right) .
$$

Let

$$
\Gamma_{p} u:=|D u|^{2} \Delta u+(p-2) \Delta_{\infty} u
$$

so that $-\Delta_{p} u \geq 0$ amounts to $-\Gamma_{p} u \geq 0$ if we do not worry about $D u=0$. We take $-\Gamma_{p} u \geq 0$ in the viscosity sense as our definition of " $p$-superharmonic". We note that this definition is consistent with the definition of " $p$-superharmonic", which employs the divergence structure of the $p$-Laplacian by virtue of the results of Juutinen, Lindqvist and Manfredi [8]. In this section we find a large family of $p$-superharmonic functions which can be built from radial functions. There are surprisingly many. Then we show that if $\mathcal{C}_{p}$ is the collection of all these functions, it is not true that $\underline{V}\left(-\Gamma_{p}\right)=\underline{S}\left(\mathcal{C}_{p}\right)$. For other interesting explicit $p$-superharmonic functions, see Lindqvist $([\overline{9}])$. 
Set

$$
\mathrm{I}_{p}^{+}:=\{\gamma \in \mathbb{R}: p+n+\gamma-4 \geq 0 \text { and } p+n+\gamma-4+(p-2)(\gamma-2) \geq 0\}
$$

and

$$
\mathrm{I}_{p}^{-}:=\{\gamma \in \mathbb{R}: p+n+\gamma-4 \leq 0 \text { and } p+n+\gamma-4+(p-2)(\gamma-2) \leq 0\} .
$$

Let us take a moment to consider these sets, each of which is a semi-infinite interval. We always assume that $p>1$. Notice that $\gamma \in \mathrm{I}_{p}^{+} \cap \mathrm{I}_{p}^{-}$requires $p+n+\gamma-4=0$ and $(p-2)(\gamma-2)=0$; hence $(p-2)(2-(n+p))=0$. Since $n+p>2$ we have

$$
\mathrm{I}_{p}^{+} \cap \mathrm{I}_{p}^{-}= \begin{cases}\emptyset & \text { if } p \neq 2 \\ \{2-n\} & \text { if } p=2 .\end{cases}
$$

It is only the case of the Laplacian when the intersection is not empty, and then the intersection is the exponent of the fundamental solution of the Laplacian. More generally, at the boundary $p+n+\gamma-4+(p-2)(\gamma-2)=0$ we have

$$
\gamma=\frac{p-n}{p-1}
$$

which, in the case $p \neq n$, is the well-known exponent for which $|x|^{\gamma}$ satisfies $\Gamma_{p}|x|^{\gamma}=$ 0 . Of course, if $p=n, \ln (|x|)$ is the radial solution.

Then define $\Phi_{\gamma}$ for $\gamma \in \mathrm{I}_{p}^{+} \cup \mathrm{I}_{p}^{-}$by

$$
\Phi_{\gamma}(x)= \begin{cases}\frac{\mathrm{s}(\gamma)}{\gamma}|x|^{\gamma} & \text { if } \gamma \neq 0 \\ \mathrm{~s}(\gamma) \ln (|\mathrm{x}|) & \text { if } \gamma=0\end{cases}
$$

where

$$
\mathrm{s}(\gamma)= \begin{cases}-1 & \text { if } \gamma \in \mathrm{I}_{p}^{+} \\ 1 & \text { if } \gamma \in \mathrm{I}_{p}^{-}\end{cases}
$$

We take $\operatorname{dom}\left(\Phi_{\gamma}\right)$ to be $\mathbb{R}^{n}$ with the origin deleted. As noted above, the definition (3.4) is only ambiguous if $p=2$ and $\gamma=2-n$, and then by linearity of $\Delta$ it does not matter below what value we assign to $\mathrm{s}(\gamma)$.

We form other functions from the $\Phi_{\gamma}$ as follows: choose $z^{i}, c \in \mathbb{R}^{n}, a_{i} \in \mathbb{R}$, $i=1, \ldots, N$, and put

$$
\begin{gathered}
\Psi(x)=\sum_{i=1}^{N} a_{i} \Phi_{\gamma_{i}}\left(x-z^{i}\right), \\
\Lambda(x)=\langle c, x\rangle+\Psi(x)
\end{gathered}
$$

where $\operatorname{dom}(\Psi)=\operatorname{dom}(\Lambda)$ is understood as $\mathbb{R}^{n}$ with $\left\{z^{1}, \ldots, z^{N}\right\}$ deleted.

The following theorem states that if $a_{i} \geq 0$ for all $i$, then $\Lambda$ is $p$-superharmonic. We invite the reader to attempt the proof of this elementary result before reading any further. It took quite some doing for us to arrive at the statement and the proof.

Theorem 3.1. Let $\Lambda$ be given by (3.6) where $p>1$. If $\gamma_{i} \in \mathrm{I}_{p}^{+} \cup \mathrm{I}_{p}^{-}$and $a_{i} \geq 0$ for $i=1, \ldots, N$, then $\Lambda$ is p-superharmonic on $\operatorname{dom}(\Lambda)$. 
Remark 3.2. Let us straightaway explain why the entire class $\mathcal{C}_{p}$ of $p$-superharmonic functions identified in Theorem 3.1 has the property $\underline{\mathrm{S}}\left(\mathcal{C}_{p}\right) \not \subset \underline{\mathrm{V}}\left(-\Gamma_{p}\right)$. Notice that if $\gamma \in I_{p}^{+}$(respectively, $\gamma \in I_{p}^{-}$), then also $\gamma \in I_{2}^{+}$(respectively, $\gamma \in I_{2}^{-}$). This follows at once from the definitions. Hence every function in $\mathcal{C}_{p}$ is 2 -superharmonic and $\underline{\mathrm{S}}\left(\mathcal{C}_{p}\right)$ contains all 2-subharmonic functions. Of course there are 2 -subharmonic functions that are not $p$-subharmonic if $p \neq 2$. For example, if $p>2$, take

$$
0<\delta<(n-1)(p-2)
$$

and put

$$
w(x)=x_{1}-\frac{n-1+\delta}{2(p-1)} x_{1}^{2}+\frac{1}{2}\left(x_{2}^{2}+\cdots+x_{n}^{2}\right) .
$$

By direct computation,

$$
-\Delta w(0)=\frac{(n-1)(2-p)+\delta}{p-1}<0, \quad-\Gamma_{p} w(0)=\delta>0 .
$$

If $1<p<2$, a modification of (3.8) provides an example.

Remark 3.3. As pointed out by P. Lindqvist, since $\Lambda$ as given in (3.6) is $p$-superharmonic and may be regarded as a sum approximating an integral, certain integrals of the form $\int|x-z|^{\gamma} d \mu(z, \gamma)$ are then automatically $p$-superharmonic. Here $\mu$ is a suitable signed measure in $\mathbb{R}^{n+1}$. More generally, any function that is the local uniform limit of $\Lambda$ 's is $p$-superharmonic.

Theorem 3.1 follows at once from the following proposition. Define

$$
Q(X, Y)_{l m}=\frac{1}{\sqrt{2}}\left(X_{l} Y_{m}-Y_{l} X_{m}\right) \text { for } X, Y \in \mathbb{R}^{n}, l, m=1, \ldots, n .
$$

It will also be convenient to rewrite $\Psi(x)$ in the form

$$
\Psi(x)=\sum_{i=1}^{N} a_{i} \Phi_{\gamma_{i}}\left(X^{i}\right)
$$

where $X^{i}=x-z^{i}$.

Proposition 3.4. Let $\Lambda(x)$ be given by (3.6), $p>1, \Gamma_{p}$ be given by (3.1) and $X^{i}=x-z^{i}$. Then

$$
\begin{aligned}
\Gamma_{p} \Lambda(x) & =\sum_{k=1}^{N} b_{k} \alpha_{k} \sum_{m, l=1}^{n}\left(Q\left(c, \hat{X}^{k}\right)_{l m}+\sum_{i=1}^{N} b_{i} Q\left(X^{i}, \hat{X}^{k}\right)_{l m}\right)^{2} \\
& +\sum_{k=1}^{N} b_{k}\left(\alpha_{k}+\beta_{k}\right)\left(\left\langle c, \hat{X}^{k}\right\rangle+\sum_{i=1}^{N} b_{i}\left\langle X^{i}, \hat{X}^{k}\right\rangle\right)^{2}
\end{aligned}
$$

where $\hat{X}_{k}=X^{k} /\left|X^{k}\right|$ and

$$
\alpha_{k}=n+\gamma_{k}+p-4, \beta_{k}=(p-2)\left(\gamma_{k}-2\right), \text { and } b_{k}=a_{k} \mathrm{~s}\left(\gamma_{k}\right)\left|X^{k}\right|^{\gamma_{k}-2} \text {. }
$$

We first prove Theorem 3.1 assuming Proposition 3.4. Finally, we turn to the proof of the proposition. 
Proof of Theorem [3.1. Notice that $\gamma_{i} \in \mathrm{I}_{p}^{+} \cup \mathrm{I}_{p}^{-}$and $a_{i} \geq 0$ together with the definition (3.4) of $\mathrm{s}(\gamma)$ imply

$$
b_{i} \alpha_{i} \leq 0 \text { and } b_{i}\left(\alpha_{i}+\beta_{i}\right) \leq 0 \text { for } i=1, \ldots, N \text {. }
$$

We conclude $-\Gamma_{p} \Psi \geq 0$, which completes the proof. Note also that all these quantities vanish if $p=2, \gamma=2-n$, no matter the value of $\mathrm{s}(\gamma)$.

We use the following lemma in the proof of Proposition 3.4

Lemma 3.5. Let $X, Y, Z \in \mathbb{R}^{n}$. Then

$$
\langle Z, Z\rangle\langle X, Y\rangle-\langle X, Z\rangle\langle Y, Z\rangle=\sum_{m, l=1}^{n} Q(Y, Z)_{l m} Q(X, Z)_{l m}
$$

where $Q$ is given by (3.9).

Proof. By direct computation,

$$
\begin{aligned}
\langle Z, Z\rangle\langle X, Y\rangle & -\langle X, Z\rangle\langle Y, Z\rangle \\
& =\sum_{m=1}^{n} Z_{m} Z_{m} \sum_{l=1}^{n} X_{l} Y_{l}-\sum_{m=1}^{n} X_{m} Z_{m} \sum_{l=1}^{n} Y_{l} Z_{l} \\
& =\sum_{m, l=1}^{n}\left(Z_{m} Z_{m} X_{l} Y_{l}-X_{m} Z_{m} Y_{l} Z_{l}\right) \\
& =\sum_{m, l=1}^{n} Z_{m} Y_{l}\left(X_{l} Z_{m}-Z_{l} X_{m}\right) \\
& =\sqrt{2} \sum_{m, l=1}^{n} Z_{m} Y_{l} Q(X, Z)_{l m} .
\end{aligned}
$$

On the other hand, interchanging the indices $l, m$ in the final sum in (3.14) does not change the value. Since $Q(X, Z)_{l m}$ is antisymmetric in $l, m$, we conclude as well that

$$
\langle Z, Z\rangle\langle X, Y\rangle-\langle X, Z\rangle\langle Y, Z\rangle=-\sqrt{2} \sum_{m, l=1}^{n} Z_{l} Y_{m} Q(X, Z)_{l m}
$$

Averaging (3.14) and (3.15) yields

$$
\langle Z, Z\rangle\langle X, Y\rangle-\langle X, Z\rangle\langle Y, Z\rangle=\sum_{m, l=1}^{n} Q(Y, Z)_{l m} Q(X, Z)_{l m}
$$


Proof of Proposition 3.4. We have, whether $\gamma$ vanishes or not,

$$
\begin{gathered}
D \Phi_{\gamma}(x)=\mathrm{s}(\gamma)|x|^{\gamma-2} x, \\
D^{2} \Phi_{\gamma}(x)=\mathrm{s}(\gamma)\left(|x|^{\gamma-2} I+(\gamma-2)|x|^{\gamma-4} x \otimes x\right)
\end{gathered}
$$

and

$$
\Delta \Phi_{\gamma}(x)=\mathrm{s}(\gamma)(n+\gamma-2)|x|^{\gamma-2}
$$

We first establish the formula

$$
\begin{aligned}
\Gamma_{p} \Psi(x)=\sum_{k=1}^{N} b_{k} \alpha_{k} \sum_{m, l=1}^{n}\left(\sum_{i=1}^{N} b_{i} Q\left(X^{i}, \hat{X}^{k}\right)_{l m}\right)^{2} \\
+\sum_{k=1}^{N} b_{k}\left(\alpha_{k}+\beta_{k}\right)\left(\sum_{i=1}^{N} b_{i}\left\langle X^{i}, \hat{X}^{k}\right\rangle\right)^{2},
\end{aligned}
$$

which is (3.10) in the case $c=0$.

For the first term in $\Gamma_{p} \Psi$, using the above we compute

$$
\begin{aligned}
|D \Psi(x)|^{2} \Delta \Psi(x) & =\left\langle\sum_{i=1}^{N} a_{i} D \Phi_{\gamma_{i}}\left(X^{i}\right), \sum_{j=1}^{N} a_{j} D \Phi_{\gamma_{j}}\left(X^{j}\right)\right\rangle \sum_{k=1}^{N} a_{k} \Delta \Phi_{\gamma_{k}}\left(X^{k}\right) \\
& =\sum_{i, j, k=1}^{N} a_{i} a_{j} a_{k}\left\langle D \Phi_{\gamma_{i}}\left(X^{i}\right), D \Phi_{\gamma_{j}}\left(X^{j}\right)\right\rangle \Delta \Phi_{\gamma_{k}}\left(X^{k}\right) \\
& =\sum_{i, j, k=1}^{N}\left(n+\gamma_{k}-2\right) a_{i} a_{j} a_{k} \mathrm{~s}\left(\gamma_{i}\right) \mathrm{s}\left(\gamma_{j}\right) \mathrm{s}\left(\gamma_{k}\right) \\
& \bullet\left|X^{i}\right|^{\gamma_{i}-2}\left|X^{j}\right|^{\gamma_{j}-2}\left|X^{k}\right|^{\gamma_{k}-2}\left\langle X^{i}, X^{j}\right\rangle \\
& =\sum_{i, j, k=1}^{N}\left(n+\gamma_{k}-2\right) b_{i} b_{j} b_{k}\left\langle X^{i}, X^{j}\right\rangle .
\end{aligned}
$$


Next we compute

$$
\begin{aligned}
& \Delta_{\infty} \Psi=\operatorname{trace}\left[(D \Psi \otimes D \Psi) D^{2} \Psi\right] \\
& =\operatorname{trace}\left[\left(\sum_{i=1}^{N} a_{i} D \Phi_{\gamma_{i}}\left(X^{i}\right) \otimes \sum_{j=1}^{N} a_{j} D \Phi_{\gamma_{j}}\left(X^{j}\right)\right)\right. \\
& \left.\bullet\left(\sum_{k=1}^{N} a_{k} \mathrm{~s}\left(\gamma_{k}\right)\left(\left|X^{k}\right|^{\gamma_{k}-2} I+\left(\gamma_{k}-2\right)\left|X^{k}\right|^{\gamma_{k}-4} X^{k} \otimes X^{k}\right)\right)\right] \\
& =\operatorname{trace}\left[\left(\sum_{i=1}^{N} a_{i} D \Phi_{\gamma_{i}}\left(X^{i}\right) \otimes \sum_{j=1}^{N} a_{j} D \Phi_{\gamma_{j}}\left(X^{j}\right)\right)\left(\sum_{k=1}^{N} b_{k} I\right)\right] \\
& +\operatorname{trace}\left[\left(\sum_{i=1}^{N} a_{i} D \Phi_{\gamma_{i}}\left(X^{i}\right) \otimes \sum_{j=1}^{N} a_{j} D \Phi_{\gamma_{j}}\left(X^{j}\right)\right)\right. \\
& \text { • } \left.\left(\sum_{k=1}^{N}\left(\gamma_{k}-2\right) b_{k} \hat{X}^{k} \otimes \hat{X}^{k}\right)\right] \\
& =\operatorname{trace}\left[\sum_{i, j, k=1}^{N} b_{i} b_{j} b_{k} X^{i} \otimes X^{j}\right] \\
& +\operatorname{trace}\left[\sum_{i, j, k=1}^{N}\left(\gamma_{k}-2\right) b_{i} b_{j} b_{k}\left(X^{i} \otimes X^{j}\right)\left(\hat{X}^{k} \otimes \hat{X}^{k}\right)\right] \\
& (3.18)=\sum_{i, j, k=1}^{N} b_{i} b_{j} b_{k}\left\langle X^{i}, X^{j}\right\rangle \\
& +\sum_{i, j, k=1}^{N}\left(\gamma_{k}-2\right) b_{i} b_{j} W b_{k}\left\langle X^{i}, \hat{X}^{k}\right\rangle\left\langle X^{j}, \hat{X}^{k}\right\rangle .
\end{aligned}
$$

Combining (3.17) and 3.18 and using Lemma 3.5, we end up with

$$
\begin{aligned}
\Gamma_{p} \Psi(x)= & |D \Psi(x)|^{2} \Delta \Psi(x)+(p-2) \Delta_{\infty} \Psi(x) \\
= & \sum_{i, j, k=1}^{N}\left(n+\gamma_{k}-2\right) b_{i} b_{j} b_{k}\left\langle X^{i}, X^{j}\right\rangle+(p-2) \sum_{i, j, k=1}^{N} b_{i} b_{j} b_{k}\left\langle X^{i}, X^{j}\right\rangle \\
& \quad+(p-2) \sum_{i, j, k=1}^{N}\left(\gamma_{k}-2\right) b_{i} b_{j} b_{k}\left\langle X^{i}, \hat{X}^{k}\right\rangle\left\langle X^{j}, \hat{X}^{k}\right\rangle \\
= & \sum_{i, j, k=1}^{N} \alpha_{k} b_{i} b_{j} b_{k}\left\langle\hat{X}^{k}, \hat{X}^{k}\right\rangle\left\langle X^{i}, X^{j}\right\rangle+\sum_{i, j, k=1}^{N} \beta_{k} b_{i} b_{j} b_{k}\left\langle X^{i}, \hat{X}^{k}\right\rangle\left\langle X^{j}, \hat{X}^{k}\right\rangle
\end{aligned}
$$




$$
\begin{aligned}
& =\sum_{i, j, k=1}^{N} \alpha_{k} b_{i} b_{j} b_{k}\left(\left\langle\hat{X}^{k}, \hat{X}^{k}\right\rangle\left\langle X^{i}, X^{j}\right\rangle-\left\langle X^{i}, \hat{X}^{k}\right\rangle\left\langle X^{j}, \hat{X}^{k}\right\rangle\right) \\
& \quad+\sum_{i, j, k=1}^{N}\left(\alpha_{k}+\beta_{k}\right) b_{i} b_{j} b_{k}\left\langle X^{i}, \hat{X}^{k}\right\rangle\left\langle X^{j}, \hat{X}^{k}\right\rangle \\
& =\sum_{k=1}^{N} b_{k} \alpha_{k} \sum_{m, l=1}^{n}\left(\sum_{i=1}^{N} b_{i} Q\left(X^{i}, \hat{X}^{k}\right)_{l m}\right)^{2} \\
& \quad+\sum_{k=1}^{N} b_{k}\left(\alpha_{k}+\beta_{k}\right)\left(\sum_{i=1}^{N} b_{i}\left\langle X^{i}, \hat{X}^{k}\right\rangle\right)^{2} .
\end{aligned}
$$

Now, we turn to the general case of (3.6) with $c \neq 0$. For the first term in $\Gamma_{p} \Lambda$, we compute

$$
\begin{aligned}
|D \Lambda(x)|^{2} \Delta \Lambda(x) & =\langle c+D \Psi(x), c+D \Psi(x)\rangle \Delta \Psi \\
& =(\langle c, c\rangle+2\langle c, D \Psi(x)\rangle+\langle D \Psi(x), D \Psi(x)\rangle) \Delta \Psi(x) \\
& =\langle c, c\rangle \Delta \Psi(x)+2\langle c, D \Psi(x)\rangle \Delta \Psi(x)+|D \Psi(x)|^{2} \Delta \Psi(x) .
\end{aligned}
$$

Next we compute

$$
\begin{aligned}
& \begin{aligned}
\Delta_{\infty} \Lambda & =\operatorname{trace}\left[((c+D \Psi) \otimes(c+D \Psi)) D^{2} \Psi\right] \\
& =\operatorname{trace}\left[(c \otimes c+c \otimes D \Psi+D \Psi \otimes c+D \Psi \otimes D \Psi) D^{2} \Psi\right]
\end{aligned} \\
& \quad=\operatorname{trace}\left((c \otimes c) D^{2} \Psi\right)+2 \operatorname{trace}\left((c \otimes D \Psi) D^{2} \Psi\right)+\operatorname{trace}\left((D \Psi \otimes D \Psi) D^{2} \Psi\right) .
\end{aligned}
$$

Combining (3.20) and (3.21) yields

$$
\Gamma_{p} \Lambda=I_{1}+2 I_{2}+I_{3}
$$

where

$$
\begin{aligned}
I_{1} & =|c|^{2} \Delta \Psi+(p-2) \operatorname{trace}\left((c \otimes c) D^{2} \Psi\right) \\
& =\sum_{k=1}^{N} b_{k}\left(n+\gamma_{k}-2\right)\langle c, c\rangle+(p-2)\left(\sum_{k=1}^{N} b_{k}\langle c, c\rangle+\sum_{k=1}^{N} b_{k}\left(\gamma_{k}-2\right)\left|\left\langle c, \hat{X}^{k}\right\rangle\right|^{2}\right) \\
& =\sum_{k=1}^{N} b_{k} \alpha_{k}\langle c, c\rangle+\sum_{k=1}^{N} b_{k} \beta_{k}\left|\left\langle c, \hat{X}^{k}\right\rangle\right|^{2} \\
& =\sum_{k=1}^{N} b_{k} \alpha_{k} \sum_{m, l=1}^{n} Q\left(c, \hat{X}^{k}\right)_{l m}^{2}+\sum_{k=1}^{N} b_{k}\left(\alpha_{k}+\beta_{k}\right)\left|\left\langle c, \hat{X}^{k}\right\rangle\right|^{2},
\end{aligned}
$$




$$
\begin{aligned}
I_{2}= & \langle c, D \Psi\rangle \Delta \Psi+(p-2) \operatorname{trace}\left((c \otimes D \Psi) D^{2} \Psi\right) \\
= & \sum_{i, k=1}^{N} b_{i} b_{k}\left(n+\gamma_{k}-2\right)\left\langle c, X^{i}\right\rangle+(p-2) \sum_{i, k=1}^{N} b_{i} b_{k}\left\langle c, X^{i}\right\rangle \\
& \quad+\sum_{i, k=1}^{N} b_{i} b_{k}(p-2)\left(\gamma_{k}-2\right)\left\langle c, \hat{X}^{k}\right\rangle\left\langle X^{i}, \hat{X}^{k}\right\rangle \\
= & \sum_{i, k=1}^{N} b_{i} b_{k} \alpha_{k}\left\langle c, X^{i}\right\rangle\left\langle\hat{X}^{k}, \hat{X}^{k}\right\rangle+\sum_{i, k=1}^{N} b_{i} b_{k} \beta_{k}\left\langle c, \hat{X}^{k}\right\rangle\left\langle X^{i}, \hat{X}^{k}\right\rangle \\
= & \sum_{k=1}^{N} b_{k} \alpha_{k}\left(\sum_{m, l=1}^{n}\left(Q\left(c, \hat{X}^{k}\right)_{l m} \sum_{i=1}^{N} b_{i} Q\left(X^{i}, \hat{X}^{k}\right)_{l m}\right)\right) \\
& \quad+\sum_{k=1}^{N}\left(b_{k}\left(\alpha_{k}+\beta_{k}\right)\left\langle c, \hat{X}^{k}\right\rangle \sum_{i=1}^{N} b_{i}\left\langle X^{i}, \hat{X}^{k}\right\rangle\right)
\end{aligned}
$$

and $I_{3}=\Gamma_{p} \Psi$, which we already computed. Forming the sum, we end up with (3.10) and are done.

Remark 3.6. Of course, there are other ways to rewrite the identity of Proposition 3.4, which expresses $\Gamma_{p} \Lambda$ as a sum of squares. For example, we could have arrived more quickly at the following result. Here we let

$$
\Psi(x)=\sum_{i=1}^{N} \frac{a_{i}}{\gamma_{i}}\left|X^{i}\right|^{\gamma_{i}}
$$

with the usual proviso in the case $\gamma_{i}=0$. For the moment, the $\gamma_{i}, a_{i}$ are arbitrary. The next computation begins as does (3.19) but concludes differently. Here $b_{i}=$ $a_{i}\left|X^{i}\right|^{\gamma_{i}-2}$.

$$
\begin{aligned}
\Gamma_{p} \Psi(x)= & |D \Psi(x)|^{2} \Delta \Psi(x)+(p-2) \Delta_{\infty} \Psi(x) \\
= & \sum_{i, j, k=1}^{N}\left(n+\gamma_{k}-2\right) b_{i} b_{j} b_{k}\left\langle X^{i}, X^{j}\right\rangle+(p-2) \sum_{i, j, k=1}^{N} b_{i} b_{j} b_{k}\left\langle X^{i}, X^{j}\right\rangle \\
& \quad+(p-2) \sum_{i, j, k=1}^{N}\left(\gamma_{k}-2\right) b_{i} b_{j} b_{k}\left\langle X^{i}, \hat{X}^{k}\right\rangle\left\langle X^{j}, \hat{X}^{k}\right\rangle \\
= & \sum_{i, j, k=1}^{N} \alpha_{k} b_{i} b_{j} b_{k}\left\langle X^{i}, X^{j}\right\rangle \\
& \quad+\sum_{i, j, k=1}^{N} \beta_{k} b_{i} b_{j} b_{k}\left\langle X^{i}, \hat{X}^{k}\right\rangle\left\langle X^{j}, \hat{X}^{k}\right\rangle
\end{aligned}
$$




$$
\begin{gathered}
=\sum_{k=1}^{N} \alpha_{k} b_{k}\left|\sum_{i=1}^{N} b_{i} X^{i}\right|^{2}+\sum_{k=1}^{N} \beta_{k} b_{k}\left\langle\sum_{i=1}^{N} b_{i} X^{i}, \hat{X}^{k}\right\rangle^{2} \\
=\sum_{k=1}^{N} b_{k} \alpha_{k}\left(\left|\sum_{i=1}^{N} b_{i} X^{i}\right|^{2}-\left\langle\sum_{i=1}^{N} b_{i} X^{i}, \hat{X}^{k}\right\rangle^{2}\right) \\
\quad+\sum_{k=1}^{N} b_{k}\left(\alpha_{k}+\beta_{k}\right)\left\langle\sum_{i=1}^{N} b_{i} X^{i}, \hat{X}^{k}\right\rangle^{2} .
\end{gathered}
$$

It is again clear that if all the coefficients $b_{k} \alpha_{k}, b_{k}\left(\alpha_{k}+\beta_{k}\right)$ have the same sign, then $\Gamma_{p} \Psi$ also has this sign.

Suppose now that $-\Gamma_{p} \Psi \geq 0$ for any choice of "poles" $z^{i}\left(\right.$ recall $\left.X^{i}=x-z^{i}\right)$. Then the $X^{i}$ can be assigned any nonzero values. Suppose that $X^{i}=r_{i} \hat{X}$ where $r_{i}>0$ for $i=1,2, \ldots, N$ and $\hat{X}$ is some unit vector. Then $b_{i}=r_{i}^{\gamma_{i}-2} a_{i}$ and

$$
\left|\sum_{i=1}^{N} b_{i} X^{i}\right|^{2}=\left\langle\sum_{i=1}^{N} b_{i} X^{i}, \hat{X}^{k}\right\rangle^{2}=\left(\sum_{i=1}^{N} a_{i} r_{i}^{\gamma_{i}-1}\right)^{2} .
$$

Hence the identity above yields

$$
-\Gamma_{p} \Psi=\left(\sum_{k=1}^{N} a_{k} r_{k}^{\gamma_{k}-2}\left(\alpha_{k}+\beta_{k}\right)\right)\left(\sum_{i=1}^{N} a_{i} r_{i}^{\gamma_{i}-1}\right)^{2} \geq 0 .
$$

Since the $r_{i}>0$ are arbitrary, this can only be if

$$
\gamma_{k} \neq 2 \Longrightarrow a_{k}\left(\alpha_{k}+\beta_{k}\right) \geq 0 \text { and } \sum_{\gamma_{k}=2} a_{k}\left(\alpha_{k}+\beta_{k}\right) \geq 0 .
$$

The condition (3.23) is necessary for $\Psi$ to be a $p$-supersolution for any choice of poles $z^{i}$ with the $a_{i}$ fixed.

On the other hand, if $z^{i}=0$ for all $i$, then all the $X^{i}$ coincide (this is the case in which all the $r_{i}$ above coincide). We now see from (3.22) that

$$
\left(\sum_{k=1}^{N} a_{k} r^{\gamma_{k}-2}\left(\alpha_{k}+\beta_{k}\right)\right)\left(\sum_{i=1}^{N} a_{i} r^{\gamma_{i}-1}\right)^{2} \geq 0 \text { for } r>0
$$

is necessary and sufficient in order that $\Psi$ be $p$-superharmonic in this case. We may assume the $\gamma_{i}$ are distinct (otherwise combine terms). In this case, we see that

$$
\sum_{k=1}^{N} a_{k} r^{\gamma_{k}-2}\left(\alpha_{k}+\beta_{k}\right) \geq 0 \text { for } r>0
$$

is necessary and sufficient. In particular, we realize that we have not described all the possible supersolutions buildable from power functions in Theorem 3.1

\section{REFERENCES}

1. E. N. Barron, R. R. Jensen and C. Y. Wang, The Euler equation and absolute minimizers of $L^{\infty}$ functionals, Arch. Rat. Mech. Anal., 157 (2001), 255-283.

2. T. Battharchaya, E. Di Benedetto, and J. Manfredi, Limits as $p \rightarrow \infty$ of $\Delta_{p} u_{p}=f$ and related extremal problems, Rend. Sem. Mat. Unvers. Politecn. Torino., Fasciocolo Speciale (1989), Nonlinear PDE's, 15-68.

3. M. G. Crandall, An efficient derivation of the Aronsson equation, preprint. 
4. M. G. Crandall, L. C. Evans and R. Gariepy, Optimal Lipschitz Extensions and the Infinity Laplacian, Calculus Var. Partial Differential Equations 13 (2001), 123-139, DOI $10.1007 / \mathrm{s} 005260000065$.

5. M. G. Crandall, H. Ishii, and P.-L. Lions, User's guide to viscosity solutions of second-order partial differential equations, Bull. Amer. Math. Soc. 27 (1992), 1-67. MR 92j:35050

6. J. T. Heinonen, T. Kilpeläinen and O. Marten, Nonlinear Potential Theory of Degenerate Elliptic Equations, Oxford University Press, Oxford, 1993. MR 94e:31003

7. R. Jensen, Uniqueness of Lipschitz extensions minimizing the sup-norm of the gradient, Arch. Rat. Mech. Anal. 123 (1993), 51-74. MR 94g:35063

8. P. Juutinen, P. Lindqvist, and J. Manfredi, On the equivalence of viscosity solutions and weak solutions for a quasi-linear equation, SIAM J. Math. Anal. 33 (2001), 699-717.

9. P. Lindqvist, On the definition and properties of p-superharmonic functions, J. für die Reine und Angewandte Mathematik 365 (1986), 67-79. MR 87e:31011

10. P. Lindqvist, On the Growth of the solutions of the equation $\operatorname{div}\left(|\nabla u|^{p-2} \nabla u\right)=0$ in $n$ dimensional space, J. Diff. Equations 58 (1985), 307-317. MR 86k:35043

Department of Mathematics, University of California, Santa Barbara, Santa BarBARA, CALIFORNIA 93106

E-mail address: crandall@math.ucsb.edu

Department of Mathematics, University of California, Santa Barbara, Santa BarBara, CALifornia 93106

E-mail address: zjyjenny@math.ucsb.edu 